\title{
EDUCACIÓN PSIQUIÁTRICA Y COMPONENTES CULTURALES EN LA FORMACION DEL MÉDICO: PERSPECTIVAS LATINOAMERICANAS
}

\section{PSYCHIATRIC EDUCATION AND CULTURAL COMPONENTS DURING MEDICAL TRAINING: LATIN AMERICAN PERSPECTIVES}

\author{
Renato D. Alarcón ${ }^{1,2, a, b}$, Manuel Suarez-Richards ${ }^{3,4, a}$, Silvana Sarabia ${ }^{1, a}$ \\ Sección de Psiquiatría, Departamento de Ciencias Médicas y Cátedra Honorio Delgado, Universidad Peruana Cayetano Heredia. Lima, Perú. \\ Mayo Clinic College of Medicine. Rochester, Minnesota, EE.UU. \\ Departamento de Psiquiatría, Facultad de Ciencias Médicas, Universidad Nacional de La Plata. Argentina. \\ Instituto Superior de Formación de Posgrado, Asociación de Psiquiatras Argentinos. Argentina. \\ Médico cirujano; ${ }^{b}$ máster en Salud Pública. \\ Recibido: 02-07-14; Aprobado: 20-08-14
}

\begin{abstract}
RESUMEN
La educación médica ha incorporado un componente psiquiátrico, o de salud mental, de manera más consistente en el curso de las últimas décadas, merced a factores tan variados como los avances de la investigación neurobiológica, la creciente prevalencia de trastornos mentales en el escenario de la salud global, la cada vez más estrecha relación entre salud mental y salud pública, comorbilidades con cuadros médicos e impacto de fenómenos socioculturales en expresiones clínicas, diagnóstico, tratamiento, pronóstico y prevención. Basado en la adquisición de competencias nucleares y principios éticos de aceptación universal, el proceso docente examinado en este artículo postula una enseñanza sustentada en la provisión de experiencias clínicas integradas a través del acopio de información adecuada, el desarrollo de capacidades diagnósticas y sujeción a una amplia variedad de modalidades de evaluación académica de estudiantes y residentes en entrenamiento. Los componentes culturales de la educación psiquiátrica reciben mención especial, proveyéndose ejemplos de su sistemática integración con la adquisición de competencias generales. Las herramientas didácticas incluyen actividades teóricas, prácticas y de supervisión. Se presta particular atención a cómo los postulados de la moderna educación médico psiquiátrica, incluidos sus aspectos culturales y la práctica de una atención médica integral como objetivos, pueden y deben tener vigencia en los países latinoamericanos.
\end{abstract}

Palabras clave: Educación; Psiquiatría; Salud mental; Educación basada en competencias; Cultura; América Latina (fuente: DECS/BIREME).

\begin{abstract}
Medical education has incorporated psychiatric or mental health components more consistently during the last decades thanks to various factors such as: advances in neurobiological research; the increasing prevalence of mental disorders in global health; the increasingly close relationship between mental health and public health; comorbidities with medical conditions and the impact of sociocultural phenomena in clinical manifestations, diagnosis, treatment, prognosis and prevention. Based on acquisition of core competencies and ethical principles of universal acceptance, the teaching process examined in this article proposes an education based on the provision of clinical experiences integrated throughout the collection of adequate information, the development of diagnostic capabilities, and exposure to a wide variety of forms of academic assessment of students and residents in training. The cultural components of psychiatric education receive special mention; we provide examples of their systematic integration with the acquisition of general skills. The teaching tools include theoretical and applied activities and supervision. Particular attention is paid to how the principles of modern psychiatric medical education, including cultural aspects and practice of holistic health care objectives, can and should be in effect in Latin American countries.
\end{abstract}

Kew words: Education; Psychiatry; Mental health; Competency-based education; Culture; Latin America (source: MeSH/NLM).

Citar como: Alarcón RD, Suarez-Richards M, Sarabia S. Educación psiquiátrica y componentes culturales en la formación del médico: perspectivas latinoamericanas. Rev Peru Med Exp Salud Publica. 2014;31(3):557-65. 


\section{INTRODUCCIÓN}

No siempre es posible diferenciar claramente la historia de la psiquiatría como especialidad médica de la del conocimiento médico en relación con las enfermedades mentales generado durante los orígenes de la medicina como ciencia en la Grecia antigua. Desde la Edad Media, el cuidado de los pacientes con trastornos mentales tenía lugar en los viejos asilos en los que el rol del médico estaba limitado al tratamiento de enfermedades físicas concomitantes. Fueron Pinel (1801) y Esquirol (1838) con sus históricas acciones y publicaciones los que, en cierto modo, dieron inicio a la psiquiatría como especialidad médica ${ }^{(1)}$. Desde una perspectiva occidental, la historia de esta última ha conocido diversas eras tales como la psicodinámica, la sociocomunitaria y la biológica ${ }^{(2)}$. A comienzos de los años 50 , la aparición de los psicofármacos dio un gran impulso a la perspectiva biológica generando cambios drásticos en la concepción y el modelo de enfermedad mental y proveyendo bases significativas para el avance contemporáneo de las neurociencias ${ }^{(3)}$. Es obvio que todos estos cambios se han reflejado, a su vez, en la educación médico-psiquiátrica, y por lo tanto, en su enseñanza.

En este contexto, la educación médica a lo largo de su historia, ha lidiado con su contraparte psiquiátrica (más ampliamente conocida como salud mental) de una manera ambivalente y hasta contradictoria. Con razón o sin ella, los argumentos predominantes han incluido en épocas diferentes, el carácter "periférico" de los temas psiquiátricos vis-a-vis el conocimiento médico convencional, su falta de basamento "científico", su carácter esencialmente "teórico" o lo que se consideraba como escasa prevalencia de las enfermedades mentales. Gradualmente, sin embargo, este enfoque ha ido variando en función de nuevos aportes de la investigación, las implicaciones pronósticas de cuadros clínicos de inicio más temprano que el tradicionalmente aceptado, las innegables interacciones entre manifestaciones anormales de salud física y salud mental (4) y documentación elocuente de una creciente relevancia de trastornos mentales en el panorama general de la salud pública. La comprobación de que varios cuadros psiquiátricos se hallan entre los de mayor impacto en índices de productividad económica, discapacidad laboral o calidad de vida ${ }^{(5)}$ ha contribuido decisivamente a un cambio sustancial en este terreno.

Es un hecho que la vinculación entre salud pública y salud mental es hoy más fuerte que nunca. En el campo educativo, más concretamente en el de la enseñanza de temas psiquiátricos a estudiantes de medicina, residentes de psiquiatría y otras especialidades, a candidatos en áreas de posgrado (becarios o fellows) y aun a médicos o psiquiatras en ejercicio, esta relación se refuerza aun más con las innovaciones en la práctica clínica actual, contextualizadas en el ejercicio de la llamada medicina (o atención) integrada ${ }^{(6)}$. El trabajo clínico a cargo de equipos multidisciplinarios, la creciente participación de pacientes, familiares, comunidades y otras fuentes de apoyo social en decisiones de manejo terapéutico, el uso cada vez más frecuente de recursos tecnológicos (desde la administración de pruebas novedosas y complicadas hasta el uso de telemedicina y telepsiquiatría) en aras de un mejor servicio, plantean nuevos e ineludibles desafíos docentes ${ }^{(7)}$.

El presente artículo intenta examinar el estado actual de la educación psiquiátrica en el mundo, como breve pero necesario antecedente de lo que se hace en América Latina, con cierto énfasis en las realidades de Perú y Argentina. Luego de un background de información epidemiológica pertinente, se procederá a una descripción de consideraciones generales y características específicas de principios y prácticas docentes en Psiquiatría y salud mental. Sobre la base de principios éticos y competencias nucleares se expondrán, en algún detalle, temas de educación psiquiátrica de pre y posgrado y sus correspondientes procesos evaluativos. Last but not least, una auténtica educación integral se beneficiará de un enfoque cultural que confiera base y secuencia armónicas, es decir, competencia cultural suficiente a estudiantes, en principio ya familiarizados con la adquisición de competencias generales en los tres dominios tradicionales del proceso didáctico: conocimientos, actitudes y destrezas. Las conclusiones precisarán el rol de la Psiquiatría en el contexto de la salud pública y la medicina integrada, sus implicaciones en lo que puede constituirse en un nuevo sistema de salud para el siglo XXI y su vigencia en la realidad actual de la educación médica latinoamericana.

\section{¿POR QUÉ ENSEÑAR PSIQUIATRÍA Y SALUD MENTAL EN MEDICINA?}

Varios autores han definido la relación entre educación médica y educación psiquiátrica como "dilemática", presentando ejemplos de diversa índole. Algunos de ellos son esencialmente dicotomías conceptuales y prácticas tales como Neurociencias vs. Ciencias Sociales, mente vs. cerebro, ciencia vs. humanismo, medicina narrativa vs. tecnología (8); otros se refieren a aspectos conflictivos tales como los de etiología y patogénesis, comorbilidades, factores de severidad o afrontes diagnósticos. Se ha puntualizado arriba, sin embargo, que en el momento actual, la educación psiquiátrica es crítica en la formación de todo médico. El 
porqué de esta afirmación proviene de diversas fuentes, pero las más elocuentes son, sin duda, las de naturaleza epidemiológica-estadística:

- Más de 500 millones de personas en el mundo sufren de trastornos mentales (y/o neuropsiquiátricos).

- Depresión mayor es la causa más importante de discapacidad a nivel global y la cuarta en la carga global de enfermedad.

- Setenta millones de personas sufren de dependencia al alcohol; al mismo tiempo, el alcoholismo está vinculado con más de 200 otras condiciones médicas.

- Veinticuatro millones tienen diagnóstico de esquizofrenia.

- Un millón al año comete suicidio y 10-20 millones lo intentan.

- Una de cada 14 personas será afectada por un trastorno mental en algún momento de su vida.

La carga mundial de morbilidad examinada de 1990 al 2010 en 291 enfermedades ${ }^{(9)}$ demostró, en 187 de ellas, que los años de vida ajustados por discapacidad (AVAD) para trastornos mentales y abuso de sustancias, constituyeron el 7,4\%; examinados individualmente, $40,5 \%$ de ellos eran trastornos depresivos, $14,6 \%$, trastornos ansiosos, consumo de drogas ilícitas 10,9\%, consumo de alcohol $9,6 \%$, esquizofrenia $7,4 \%$, trastorno bipolar $7,0 \%$, trastorno generalizado del desarrollo $4,2 \%$, trastorno de comportamiento en la infancia 3,4\% y trastornos de la alimentación, $1,2 \%$. Finalmente, $25 \%$ de pacientes vistos en clínicas de atención primaria (AP) tienen un trastorno mental añadido, un $88 \%$ de pacientes mentales buscan primero cuidados en AP y en la mitad de los casos de depresión (y más en trastornos alimentarios o alcoholismo), el diagnóstico inicial es erróneo.

Cuando se habla de comorbilidad, uno de los ejemplos más citados es el de depresión como diagnóstico concomitante en $32,5 \%$ de pacientes con diabetes, $32 \%$ en aquellos con historia previa de accidente cerebrovascular, $25 \%$ en pacientes con cáncer, $16 \%$ en quienes han tenido infarto miocárdico y $10-20 \%$ de mujeres en fase posparto. Se ha demostrado, también, el impacto agravante de depresión en una variedad de cuadros, particularmente de naturaleza cardiovascular, respiratoria y endocrinológica ${ }^{(10)}$.

Es obvio que las demandas de atención y cuidado de estos problemas de salud superan las posibilidades de la actual fuerza de trabajo constituida por psiquiatras y otros profesionales de salud mental. África, cuenta solo con 0,4 psiquiatras por 100000 habitantes frente a Europa con 9,8; Asia tiene 0,1 enfermeras psiquiátricas por 100000 habitantes frente a Europa con 24,8 . Solo poco más de la mitad de pacientes con depresión grave o ansiedad generalizada (56,3 y $57 \%$, respectivamente) reciben tratamiento más 0 menos adecuado; esta cifra desciende a $32,2 \%$ en el caso de pacientes esquizofrénicos y sube a 78,1 en pacientes con alcoholismo, aunque la duración de sobriedad en estos últimos fluctúa significativamente ${ }^{(9,10)}$. El suicidio es una de las tres principales causas de muerte en personas de 15 a 34 años (en su mayoría, varones), al lado de accidentes y cáncer. La necesidad de formar más y mejores psiquiatras a nivel mundial es imperativa.

\section{LA EDUCACIÓN PSIQUIÁTRICA EN EL MUNDO}

De todas las ramas de la Medicina, la Psiquiatría, se constituyen en amalgama o vertiente final en la conjunción de ciencias naturales y ciencias humanas. Es probable que los cambios científicos, las influencias tecnológicas, sociales, de mercado y financieras, modificarán los estudios, la educación y consecuentemente la práctica psiquiátrica del siglo XXI, pero aun así, se estima que aquella convergencia de conocimientos ha de perdurar $y$, con ella, una indetenible combinación de ideas, de procesos y tecnologías que permiten dar herramientas a la teoría y teoría a las herramientas ${ }^{(11)}$. Es pues indispensable anticipar en la medida de lo posible las tendencias e innovaciones del mañana con la finalidad de plasmar lo que hoy en día es la proclama y el postulado fundamental de la educación en nuestro campo: la formación integral del psiquiatra.

Se ha señalado ya que la educación médica actual ha solidificado su vinculación a inequívocos principios éticos (11). En síntesis, ellos son: a) Aceptación de la identidad propia (del profesional en formación); b) Validación del "otro" (el paciente y sus relacionados); c) Vigencia de reconocimiento e inclusión; d) Capacidad de escuchar; e) Imparcialidad y objetividad; f) Independencia; g) Comprensión y empatía; h) Beneficio de la duda como elemento de convalidación y mejora; i) Aceptación de normas universalmente sancionadas; j) Honestidad, y k) Protección de la seguridad propia y ajena. Por otro lado, la educación médica es un proceso de desarrollo desigual en diferentes regiones del mundo. A su complejidad conceptual se unen las distinciones en cuanto a tipos de fuerza laboral docente y los costos y presupuestos que su presencia y su vigencia requieren. Europa y Norteamérica parecen poseer estructuras e instrumentos más maduros y flexibles, de aplicabilidad más o menos amplia y coherente. Tal no parece ser el caso en los otros continentes, con la posible excepción 
de Oceanía que se acerca más al modelo anglosajón. En general, puede decirse que la educación médica a nivel global confronta dos tipos de desafío, uno vinculado con aspectos logísticos, y otro con aspectos técnicos. Dentro de aquellos se cuentan, por cierto, enseñanza y aprendizaje como actividades en necesidad de implementaciones concretas, contenidos curriculares (dictados por la diversidad de "profesiones de la salud"), estilos pedagógicos y modalidades docentes; los aspectos técnicos, se refieren a niveles de enseñanza (estudiantes, residentes, fellows, etc.) y temas de syllabi: básicos, clínicos, nosológicodiagnósticos o culturales, para citar algunos ${ }^{(12)}$.

Las competencias básicas se hallan bien delineadas en dos importantes documentos publicitados por entidades académico-docentes de los Estados Unidos: el American Council of Graduate Medical Education (ACGME) (13) y el Proyecto Milestone (14). Para muchos, estas competencias se resumen en lo que se conoce como profesionalismo. Este concepto incluye siete elementos de naturaleza a la vez teleológica y pragmática: puntualidad, eficiencia, límites éticos, capacidad de comunicación, cortesía, confidencialidad y respeto/empatía (11,15). Las competencias básicas pueden, a su vez, ser genéricas (por ej., conocimientos, habilidades, actitudes y buen juicio; cualidades personales engarzadas con recursos instrumentales y atributos sistémicos) y específicas (por ej., análisis crítico, apreciación de la investigación como sustento de adecuada información clínico-científica, capacidad de trabajo en equipo, acatamiento de normas legales, creatividad y liderazgo y respeto a la autonomía del paciente, a sus creencias y a su cultura ${ }^{(16)}$.

Entendidas como actuaciones integrales para identificar, interpretar, argumentar y resolver problemas del contexto clínico con idoneidad y ética, las competencias nucleares en la educación psiquiátrica entrañan la secuencia "saber-cómo hacer-demostrar cómo hacer-hacer" extendida a entidades clínicas, procedimientos diagnósticos, tratamientos, actitudes y manejo interpersonal de cada caso. Finalmente, un importantísimo componente del proceso educativo en medicina es el de la evaluación, cuyos requisitos esenciales son: validez, fiabilidad, factibilidad, aceptabilidad e impacto educativo propiamente dicho. La evaluación se da en los niveles de pregrado, posgrado y de ejercicio profesional, tiene una naturaleza eminentemente práctica y, con tal propósito en mente, utiliza, entre otros, instrumentos como entrevistas a pacientes (estandarizados o "simulados"), imágenes clínicas, cuestionarios cortos, pruebas de elección múltiple, examen oral estructurado o informes clínicos (17).

\section{LA SALUD MENTAL EN AMÉRICA LATINA}

Con unos 600 millones de habitantes, se estima que América Latina figura entre las regiones más urbanizadas, pero al mismo tiempo, más desigualmente pobladas del mundo en relación a la extensión de su territorio donde el $80 \%$ vive en ciudades, proporción superior a la de países desarrollados (18); en el año 2020 habrá en el mundo 550 ciudades con más de un millón de habitantes, casi 100 de ellas en nuestro subcontinente. Esto provoca, ya sin duda, cambios en todas las aristas personales y relacionales, individuales y colectivas, las poblaciones se modifican, las familias cambian su entramado y, con ello, sus problemas y patologías psíquicas.

El $73 \%$ de los países asignaron solo del 1 al $5 \%$ de su presupuesto sanitario a la salud mental en 2011, a pesar de específicas recomendaciones de incremento (18). Estas ineficiencias y la persistente presencia de estigma y prejuicio en diferentes esferas continúan limitando el uso de recursos públicos y privados. Las publicaciones en salud mental son escasas, únicamente $2,6 \%$ del total a nivel mundial.

En Argentina, con 41 millones de habitantes, un 92\% vive en áreas urbanas, más de seis millones (con $46 \%$ de mujeres) padecen de trastornos mentales. Se consumen altos niveles de agentes psicotrópicos y el $26 \%$ de ellos son adquiridos sin receta médica ${ }^{(19)}$. El trastorno depresivo mayor afecta a 1,6 millones (68\% mujeres); el abuso o dependencia al alcohol aqueja a 1,7 millones ( $87 \%$ varones); el consumo de alcohol per cápita (+ de 15 años) fue de 19,5 litros de alcohol puro para varones y 10,9 para mujeres, en 2010. En el Perú, estudios recientes ${ }^{(7,20)}$ han mostrado una prevalencia anual de trastornos mentales del $13,5 \%$, con más frecuencia para cuadros ansiosos $(7,9 \%)$, trastornos del ánimo y control de impulsos $(3,5 \% \mathrm{c} / \mathrm{u})$ y uso de sustancias $(1,7 \%)$. Solo $32 \%$ de pacientes con trastornos severos recibieron algún tipo de tratamiento en los últimos 12 meses, con porcentajes aun menores en casos de menor severidad. La prevalencia de vida fue del $29 \%$ y cohortes jóvenes presentaron más riesgo de experimentar una enfermedad mental.

\section{PRINCIPIOS GENERALES DE LA EDUCACIÓN PSIQUIÁTRICA}

Un primer punto de acuerdo en el campo de la educación psiquiátrica moderna a estudiantes de Medicina y a residentes en entrenamiento, es el de posibilitar una experiencia clínica integrada. Ella incluye 
sesiones clínicas, laboratorios de habilidades clínicas, simulaciones de eventos clínicos variados y pasantías programadas por centros de salud, hospitales, salas de emergencia, centros comunitarios urbanos, postas rurales y otros establecimientos asistenciales. La especialización en psiquiatría requiere una estructura suficientemente flexible. Posiblemente más que en otros campos, los postulados didácticos fundamentales de la educación psiquiátrica deben orientarse a identificar las necesidades fundamentales de cada caso individual, a trabajar en estrecha colaboración con pacientes, familiares, cuidadores, amigos y las redes de apoyo social, fomentando en paralelo el crecimiento personal y epistemológico del futuro profesional.

Dentro de sus principios generales, la educación psiquiátrica actual señala que el estudiante deberá demostrar al término de su entrenamiento, la capacidad de: a) Organizar la información clínica de manera tal que pueda elaborar posibilidades diagnósticas razonables en torno a trastornos establecidos, condiciones concomitantes y problemas psicosociales; b) Identificar las características fundamentales de las entidades psiquiátricas incluidas en nomenclaturas aceptadas; c) Formular planteamientos realistas y bien fundamentados en relación a etiopatogénesis y curso clínico de la enfermedad; d) Precisar indicaciones pertinentes para pruebas de laboratorio, interpretar e informar adecuadamente sus resultados y optimizar niveles de adherencia a tratamientos establecidos; e) Mantenerse alerta a factores de tipo cultural en juego a lo largo de la historia de vida y la historia clínica del paciente ${ }^{(12,21)}$.

Al lado de las competencias generales, otras que afectan más específicamente a la Psiquiatría incluyen: a) Conocer los principales tipos de psicoterapias; b) Conocer y manejar psicofarmacología y otras formas de intervenciones terapéuticas; c) Conocer neurología, para diagnósticos diferenciales; d) Contribuir eficazmente al manejo y la asistencia de pacientes médicos y quirúrgicos (psiquiatría de enlace); e) Trasmitir información clara a colegas, integrar equipos interdisciplinarios con ellos y otros profesionales de la salud mental; f) Conocer psiquiatría administrativa y prevalecer en el liderazgo de equipos clínicos multidisciplinarios.

Es indispensable prestar particular atención a la formación en psicoterapia, lamentablemente reducida o postergada en las últimas décadas debido, en parte, a la falacia de que "el psiquiatra prescribe medicamentos y el psicólogo habla con el paciente", así como al predominio a veces irreflexivo de las contribuciones neurobiológicas. El psiquiatra no debe renunciar a la formación y utilización de esta práctica, cualquiera que fuere la escuela u orientación a la que se adhiera.

\section{COMPONENTES CULTURALES DE LA EDUCACIÓN PSIQUIÁTRICA}

No sorprende el que en función de nuevas realidades en el campo de la llamada salud global, las distinciones culturales entre regiones, países y comunidades étnicas o tribales hagan necesaria la consideración de componentes culturales definidos en el desarrollo de programas de educación psiquiátrica. Si definimos cultura como el conjunto de factores que confieren identidad, significado y presencia a grupos humanos en cualquier lugar del planeta, factores que van desde lenguaje o religión hasta tradiciones, dieta o filosofía económico-financiera, resulta claro que su vigencia en la concepción, la experiencia y el manejo de condiciones de salud y enfermedad es poderosa ${ }^{(22)}$. Más aun, cultura y sus muchas variables cumplen un papel decisivo en la interpretación o modelos explicativos de conductas y síntomas, en la patogénesis de cuadros clínicos emocionales, en el diagnóstico y clasificación de trastornos, en su tratamiento y en la provisión misma de servicios. Numerosas organizaciones y agencias nacionales e internacionales que tienen que ver con el proceso educativo en Medicina y Psiquiatría han reconocido abiertamente estos hechos. El principal problema radica, sin embargo, en la medida en que este reconocimiento se refleja en el currículo de programas de pre y posgrado.

El ACGME, por ejemplo, señala claramente que los pacientes destinados a asistir en el entrenamiento de residentes «deben proceder de una variedad de backgrounds étnicos, raciales, socioculturales y económicos»; que es indispensable la comprensión y conocimiento de la cultura global y subculturas de los pacientes «con un foco específico en el adiestramiento de residentes con backgrounds culturales diferentes»; que la formulación de un caso clínico incluya «ítems socioculturales involucrados en su diagnóstico y manejo terapéutico» (13). En este contexto, las características fundamentales del enfoque cultural en la educación psiquiátrica entrañan consistencia (en tiempo y espacio, en contenido y dispensación), creatividad (reflejada en adaptabilidad, originalidad y conveniencias logísticas) y alcance integral (en amplitud, cobertura y profundidad).

Por espacio de casi tres años, un Comité Especial de la Asociación Psiquiátrica Mundial trabajó en un modelo de programa curricular concebido sobre la base de los dominios conocidos (conocimientos, destrezas y actitudes) y con una secuencia gradualmente 
más compleja pero coherente, tanto vertical (es decir, de estudiantes de medicina a residentes de la especialidad) como horizontal (de competencias generales a estrictamente culturales) ${ }^{(23)}$. Presentamos ejemplos puntuales de esta estructura en cada uno de los dominios.

I) Dominio: Conocimientos

Competencia general: plan integral de tratamiento

Competencias culturales vinculadas

Para estudiantes de Medicina: fomento de cuidados multidisciplinarios

Para residentes de Psiquiatría: aspectos culturales de tratamientos psicológicos y farmacológicos; psicoterapias culturales; intervenciones médicolegales.

II) Dominio: Destrezas

Competencia general: entrevista clínica con información completa resultante

Competencias culturales vinculadas

Para estudiantes de Medicina: información demográfica; exploración inicial de variables/factores culturales en el cuadro clínico

Para residentes de Psiquiatría: identidad cultural; formulación cultural.

III) Dominio: Actitudes

Competencia general: profesionalismo, empatía y comprensión

Competencias culturales vinculadas

Para estudiantes de Medicina: diferencias raciales, étnicas, sociales y culturales; historial de migración; migrantes, refugiados y minorías étnicas.

Para residentes de psiquiatría: historia familiar, jerarquías, mecanismos de afronte, puntos de vista, opiniones, etc.; entidad unitaria cuerpo-mente, perspectivas holísticas.

\section{HERRAMIENTAS DIDÁCTICAS}

El cómo enseñar constituye uno de los desafíos más grandes a los educadores psiquiátricos, a la luz de una enseñanza basada en competencias. Las herramientas didácticas se han multiplicado y su nivel de complejidad también ha crecido en función de necesidades evidentes y de avances tecnológicos reales. Trátese de competencias generales o específicas (i.e., culturales) en Medicina o en Psiquiatría, es importante tener presente una variedad de premisas básicas (24): a) La percepción sistemática del impacto multiforme de realidades bíopsicosocioculturales y espirituales en la entidad clínica bajo estudio; b) La necesidad de conocer y utilizar múltiples enfoques didácticos y ejercicios clínicos; c) El estímulo y la búsqueda consistente de feedback y aportes críticos constructivos de estudiantes y residentes y d) Uso racional de los modernos avances tecnológicos en función de las necesidades de cada etapa o nivel del proceso docente.

Las tres facetas primordiales de este proceso (conferencias o clases teóricas, actividades prácticas y tareas de supervisión) requieren herramientas didácticas especialmente adaptadas. Así por ejemplo, en el caso de la capacitación en competencias culturales, las conferencias o clases teóricas deben cubrir dimensiones y aplicaciones clínicas, formulaciones culturales (a la manera de la formulación para la entrevista cultural, recientemente introducida en el DSM-5) ${ }^{(25)}$, poblaciones especiales, narrativas "en primera persona", cultura y diagnóstico, familiarización con contribuciones clásicas de la literatura psiquiátrica y temas de investigación actual.

Las actividades prácticas, por su parte, deberán incluir: a) Utilización apropiada de diferentes clases de escenarios clínicos; b) Casos en psicoterapia, con énfasis en el uso de las llamadas "psicoterapias culturales"; c) Demostraciones individuales de aspectos especiales del trabajo en equipo, a cargo de diferentes miembros del mismo; d) Actividades complementarias y de "refuerzo" tales como revista de revistas, discusiones de películas escogidas, grupos experienciales, terapia historiográfica, psicodrama, etc. ${ }^{(13,14,26)}$.

Finalmente, la supervisión se refiere no solo a la de los estudiantes o futuros especialistas, sino a los miembros de la plana docente misma; para aquellos, y en un contexto formal o informal, individual o de grupo, el apoyo bibliográfico múltiple (desde capítulos en libros de texto hasta ensayos o memorias de pacientes o profesionales) o la novedosa técnica de discusión de "diarios" (journals) escritos por los propios estudiantes sobre sus experiencias de aprendizaje cuotidiano con autocríticas y críticas constructivas a los docentes, abren oportunidades sugerentes. Para los instructores o profesores, la provisión, de oportunidades adecuadas de entrenamiento adicional o actualizaciones de técnicas docentes es esencial (17).

La enseñanza y el aprendizaje clínicos de la psiquiatría cultural son descritos, en la terminología actual, como basados en: a) Problemas (por ej., experiencias de duelo en miembros de diferentes grupos étnicos o comunitarios); b) Evidencias (por ej., respuesta a medicaciones por parte de miembros de diferentes etnias - farmacogenómica psiquiátrica); c) Labor de equipo (por ej., presentaciones de casos a cargo de diferentes profesionales de Salud Mental), y 
d) Experiencias personales o auto biográficas de profesores y estudiantes.

\section{REALIDADES DE LA EDUCACIÓN PSIQUIÁTRICA EN AMÉRICA LATINA}

El número de psiquiatras varía marcadamente entre países latinoamericanos, con una tasa de 1,5 por 100000 habitantes para América Central, México y el Caribe; 1,9 para el Caribe no latino, y 2,9 para América del Sur. En Argentina hay 4500 psiquiatras de un total de 160 mil médicos en edad activa ${ }^{(27)}$, con una mayor concentración en las ciudades más pobladas. El déficit de especialistas en el Perú muestra cifras similares. La psiquiatría se encuentra entre las 25 especialidades y subespecialidades consideradas prioritarias para la formación de profesionales en Medicina (28). En la actualidad, hay 695 psiquiatras inscritos en el Registro Nacional de Especialistas del Colegio Médico del Perú. Esta cifra es casi tres a cuatro veces menor que la de cirujanos generales (2055), ginecoobstetras (2954), pediatras (2833) e internistas (1969) (29). Con una población que se aproxima a los 31 millones ${ }^{(30)}$, el Perú debería tener, de acuerdo a estimados de la Organización Mundial de la Salud (OMS), por lo menos un psiquiatra por cada 10000 habitantes, es decir, un mínimo de 3100 psiquiatras, por lo que el déficit actual es de aproximadamente 2400 . Además, es importante tener en cuenta que el crecimiento anual de población alcanza un promedio de $1,6 \%$, es decir casi 400000 habitantes, por lo que anualmente deberían registrarse un mínimo de 40 nuevos psiquiatras, si se trata de prevenir el continuo aumento del déficit mencionado arriba.

La diversidad de culturas, tradiciones, escuelas psiquiátricas y otros matices diferenciales promueven variados componentes curriculares formativos dentro de los países latinoamericanos. Por ejemplo, el Instituto Superior de Formación de Postgrado (ISFP) de la Asociación de Psiquiatras Argentinos (APSA) trabaja en el entrenamiento de especialistas y desarrollo de actividades de educación permanente en diferentes regiones del país donde, a pesar de la existencia de programas públicos o privados, la necesidad se percibe con mayor impacto; se ha establecido la carrera de posgrado de la CA de Buenos Aires (Centro) y carrera de especialista en Neuquén y en la Universidad del Comahue (Patagonia). A mediados del 2014 inició sus labores en este terreno, la Universidad de Tucumán (Noroeste); la Universidad del Comahue comenzará igualmente en 2015 , la carrera de especialista Infantojuvenil (hasta hoy ausente en toda la región patagónica) y la Maestría en Psiquiatría Forense. Igualmente, el ISFP conduce talleres sobre trastornos depresivos para médicos de atención primaria en todas las provincias, conjuntamente con la Facultad de Ciencias Médicas de la Universidad de La Plata (31). APSA es evaluadora formal del Sistema Nacional de Residencias del Equipo de Salud y lleva a cabo el Programa de Actualización en Psiquiatría (PROAPSI) que provee educación médica continua a distancia (SEMCAD).

EnelPerú, elaño2002, el Comité Nacional de Residentado Médico (CONAREME) definió los estándares mínimos de formación para todos los programas de segunda especialización en psiquiatría, los cuales rigen hasta la actualidad (28). El documento consta de dos partes: la primera contiene las condiciones necesarias para el logro de los estándares, y la segunda presenta los estándares mínimos propiamente tales relacionados con el perfil académico, los objetivos curriculares y los logros mínimos para cada uno de los tres años de estudio. Sobre estas bases, las siguientes instituciones académicas peruanas conducen programas aprobados de entrenamiento psiquiátrico de posgrado: Universidad Peruana Cayetano Heredia, Universidad Nacional Mayor de San Marcos, Universidad Nacional Federico Villarreal, Universidad Ricardo Palma, Universidad Particular de San Martín de Porres, Universidad Nacional Pedro Ruiz Gallo (Chiclayo), Universidad Nacional de Trujillo, Universidad Privada Antenor Orrego (Trujillo) y Universidad Nacional de San Agustín (Arequipa).

La evaluación de las competencias adquiridas, es decir el nivel de calidad de lo que ha de constituir la práctica clínica diaria ${ }^{(12,23,24)}$ se ha desplazado del clásico examen escrito que evalúa casi exclusivamente conocimientos mas no destrezas, a una detallada evaluación de todos los niveles y los componentes del entrenamiento. Los tutores o supervisores pueden usar varios métodos para optimizar la evaluación de las diversas rotaciones clínicas. Algunos de uso cada vez más sistemático incluyen la evaluación clínica propiamente tal, a cargo de expertos, el informe regular y consistente del supervisor, el portafolio de actividades y la evaluación de la progresión de competencias a lo largo del año o del periodo específico de enseñanza ${ }^{(32,33)}$.

En los últimos años se está empleando en diversos países el Objective Structured Clinical Examination o examen clínico objetivo estructurado (ECOE) que postula una evaluación planificada de las competencias clínicas, de sus componentes y desempeños. El examen consta de estaciones o etapas que conforman un circuito. En cada estación, el estudiante se enfrenta a una situación que evalúa una competencia clínica y desempeños específicos en las áreas de conocimiento, habilidades y actitudes ${ }^{(34)}$. El ECOE muestra una buena evidencia psicométrica, principalmente en alumnos de 
pregrado ${ }^{(35)}$. La experiencia con este sistema en el Perú y otros países latinoamericanos es muy limitada, a pesar de haberse empezado a utilizar en otras especialidades médicas en el mundo desde 1975, y por primera vez en psiquiatría, el año 1994 en la Universidad de Toronto. La evaluación más sofisticada, sin embargo, no disminuye la enorme relevancia de lo que llamamos automotivación del estudiante para la genuina adquisición de competencias valiosas y para un aprendizaje a fondo de la psiquiatría como ciencia y como experiencia interhumana de la más alta factura.

En un intento de favorecer un continuo avance de estos aspectos en el ámbito latinoamericano, la Sección de Educación en Psiquiatría de APAL, ha propuesto consensuar un currículo homologable para las carreras de posgrado dentro de nuestra región, con competencias similares y/o equivalentes, que posibiliten el ejercicio de la especialidad en los diferentes países que integran la organización ${ }^{(36)}$. De este modo, un título que se otorgue en un país y un entrenamiento consolidado y eficiente serían credenciales válidas en otros, generando el potencial afianzamiento de un útil flujo migrante de psiquiatras en el continente. Se trata de una iniciativa innovadora que requerirá atención y análisis cuidadosos en el camino a su implementación.

\section{CONCLUSIONES}

La formación del psiquiatra, en muchos países, ha evolucionado desde un modelo paternalista basado en la labor del "aprendiz", aquél que aprende la profesión observando a un maestro durante un lapso determinado, al más reciente en el ámbito global, basado en competencias, con metas precisas, procedimientos definidos y evaluaciones, según rigurosos estándares de acreditación. El modelo contemporáneo estipula que el alumno, más aun, el residente, deberá demostrar que ha adquirido las destrezas, que las conoce y maneja de manera eficiente y eficaz; el modelo basado en competencias no depende necesariamente del tiempo transcurrido sino en la demostración de habilidades y capacidad de actuar en conformidad con evidencias científicas y prácticas debidamente comprobadas y medidas, posibilitando un óptimo desempeño profesional.

La educación psiquiátrica constituye en la actualidad un desafío multiforme que hace necesaria una colaboración integral de pre a posgrado, con la ecuación en salud pública y salud mental como eje inquebrantable. El componente cultural debe ser parte sustancial de todo programa docente, más aun en escuelas médicas y programas de residentado psiquiátrico en países de América Latina. La misión docente en psiquiatría será así uno de los pilares fundamentales en el proceso de promoción, prevención, asistencia e investigación en salud mental. Es imperativo desarrollar programas de colaboración interinstitucional e internacional en docencia, incorporando las diferentes modalidades de una moderna atención médica integrada a lo largo del continente.

Contribuciones de autoría: RDA participó en la concepción y redacción del artículo, RDA, MSR y SS participaron en recolección y análisis de la información, MSR y SS participaron en la revisión del artículo y aprobación de la versión final.

Conflictos de interés: los autores declaran no tener conflictos de interés.

Fuente de financiamiento: autofinanciado

\section{REFERENCIAS BIBLIOGRÁFICAS}

1. Pichot P. The history of psychiatry as a medical specialty. In: Gelder MG, Andreasen N, Lopez-Ibor JJ, Geddes J, editors. New Oxford Textbook of Psychiatry. 2a ed. Oxford: Oxford University Press; 2009. p. 17-27.

2. Mora G. Historical and theoretical trends in Psychiatry. In: Freedman AM, Kaplan BJ, Sadock VA, editors. Comprehensive Textbook of Psychiatry. 3rd. ed. Vol. I. Philadelphia, PA: Lippincott Williams \& Wilkins; 1980. p. 4-98.

3. Finger S. Origins of Neuroscience. Oxford: Oxford University Press; 1994.
4. Toft T, Fink P, Oernboel E, Chistensen K, Frostholm L, Olesen F. Mental disorders in primary care: prevalence and comorbidity among disorders. Results from the functional illness in primary care (FIP) study. Psychol Med. 2005 Aug;35(8):1175-84.

5. Abegunde DO, Mathers CD, Adam T, Ortegon M, Strong K. The burden and costs of chronic diseases in low-income and middle-income countries. Lancet. 2007 Dec:370(9603):1929-38.

6. Cassel CK, Guest JA. Choosing wisely: helping physicians and patients make smart decisions about their care. JAMA. 2012 May 2;307(17):1801-2. doi: 10.1001/jama.2012.476.
7. Diez-Canseco F, Ipince A, Toyama M, Benate-Galvez Y, Galán-Rodas E, Medina-Verástegui JC ,et al. Atendiendo la salud mental de las personas con enfermedades crónicas no transmisibles en el Peru: Retos y oportunidades para la integración de cuidados en el primer nivel de atención. Rev Peru Med Exp Salud Publica. 2014;31(1):131-6.

8. Alarcón RD. La disciplina de los perennes comienzos. Dilemas de la psiquiatría contemporánea. En: Alarcón RD, Castro J, Cipriani E, editores. Desde nuestra propia entraña. Lima: Talleres Gráficos P.L. Villanueva; 1991. p. 117-143. 
9. Murray CJ, Vos T, Lozano R, Naghavi M, Flaxman AD, Michaud C, et al. Disability-adjusted life years (DALYs) for 291 diseases and injuries in 21 regions, 1990-2010: a systematic analysis for the Global Burden of Disease Study 2010. Lancet. 2012 Dec 15;380(9859):2197-223. doi: 10.1016/S0140-6736(12)61689-4.

10. Egede LE. Major depression in individuals with chronic medical disorders: prevalence, correlates and association with health resource utilization, lost productivity and functional disability. Gen Hosp Psychiatry. 2007 SepOct;29(5):409-16.

11. Suárez-Richards M. Neurociencias, Neuroética y Psiquiatría. Debates em Psiquiatría. 2012 Set-Out;2(6):6-10.

12. Harden RM, Laidlaw JM. Essential skills for a medical teacher. An Introduction to teaching and learning in medicine. Edinburgh: Churchill Livingstone; 2012.

13. American Council of General Medical Education. Core competencies in Medical Education. Washington DC: ACGME, 2007.

14. Substance Abuse and Mental Health Services Agency; American Council of General Medical Education. The Milestone Project in Medical Education. Washington DC: SAMHSA/ACGME; 2012.

15. Suárez Richards M. La formación integral del psiquiatra, Parte I. Buenos Aires: Panamericana; 2013.

16. González-Díaz C, Sánchez-Santos L. El diseño curricular por competencias en la educación médica. Educ Med Sup. 2003; 17(4):21-7.

17. Berry LL, Seltman KD. Management lessons from Mayo Clinic: inside one of the World's Most Admired Service Organizations. New York, NY: McGraw Hill; 2008.

18. Organización de las Naciones Unidas. Programa de las Naciones Unidas para los Asentamientos Humanos [Internet]. Río de Janeiro: ONU; 2012 [citado el 02 de julio del 2014]. Disponible en: http://www.nu.org.bo/agencia/ programa-de-las-naciones-unidas-paralos-asentamientos-humanos/

19. Di Filippo P. It's a Mad, Mad, Mad, World. The Argentina Independent. October 10, 2012.

20. Piazza M, Fiestas F. Prevalencia anual de trastornos y uso de servicios de salud mental en el Perú: Resultados del Estudio Mundial de Salud Mental, 2005. Rev Peru Med Exp Salud Publica. 2014 Ene-Dic;31(1):30-8.

21. Gutiérrez M. La educación médica del futuro. Bilbao: Centro de Investigación en Red de Salud mental/CIBERSAM; 2008.

22. Alarcón RD, Westermeyer J, Foulks EF, Ruiz P. Clinical relevance of contemporary cultural psychiatry. J Nerv Ment Dis. 1999 Aug;187(8):46571.

23. Tasman A, Alarcón RD, Kay J, Jenkins R. Competencies for Psychiatric Education. Report from the Special Committee of the World Psychiatric Association. Chêne-Bourg; WPA, 2012.

25. Malik A, Bhugra D, Brittlebank A. Workplace-Based Assessments in Psychiatry. 2nd ed. Londres: The Royal College of Psychiatrists; 2011.

24. Lewis-Fernández R, Aggarwal NK. Culture and psychiatric diagnosis. Adv Psychosom Med. 2013;33:15-30. doi: 10.1159/000348725.

25. Weerasekera P, Feinstein RE. The future of psychiatric education: an international perspective. Acad Psychiatry. 2013 Jul 1;37(4):229-32. doi: 10.1176/appi.ap.13030023.

26. Argentina, Ministerio de Salud de la Nación. Médicos en edad activa al 31-12-2012. Boletín de la Dirección Nacional de Regulación Sanitaria. Buenos Aires; 2013.

27. Comité Nacional de Residentado Médico [Internet]. Lima: CONAREME; 2014 [citado el 06 de junio de 2014]. Disponible en: http:// www.conareme.org.pe/

28. Colegio Médico del Perú [Internet]. Lima, Perú: ¿Conoce a su médico?:
2014. [citado el 04 de junio de 2014] Disponible en: http://www.cmp.org. pe/servicios/iconoce-a-su-medico. html

29. Instituto Nacional de Estadística e Informática [Internet]. Lima: INEI; 2014 [citado el 30 de mayo de 2014]. Disponible en: http://www.inei.gob.pe

30. Suárez Richards M, Pujol S, ArboledaFlores J, Goñi M. Estudio del conocimiento sobre los trastornos depresivos entre los médicos asistenciales en Argentina. Vertex. 2009 Nov-Dic;20(88):405-10.

31. Abdelaziz A, Koshak E. Triangular model integrating clinical teaching and assessment. Adv Med Educ Pract. 2014 Mar 4;5:61-4. doi: 10.2147/AMEP. S48789.

32. Hartley S, Gill D, Carter F, Walters K, Bryant P. Teaching Medical Students in Primary and Secondary Care: A Resource Book. Oxford, UK: Oxford University Press; 2003.

33. Hodges BD, Hollenberg E, McNaughton N, Hanson MD, Regher G. The Psychiatry OSCE: A 20-year retrospective. Acad Psychiatry. 2014 Feb;38(1):26-34. doi: 10.1007/ s40596-013-0012-8.

34. Triviño $\mathrm{X}$, Vásquez A, Mena A, López A, Aldunate M, Varas M, et al. Aplicación del examen clínico estructurado (OSCE) en la evaluación final del internado en pediatría en dos escuelas de medicina. Rev Med Chile. 2002;130:817-24.

35. Córdoba R, Suárez-Richards M. La homologación de la Curricula de Post-grado en Psiquiatría. Revista Latinoamericana de Psiquiatría. 2013;12(2):12-7.

Correspondencia: Renato Alarcón Guzmán Dirección: Casa Honorio Delgado. Av. Armendáriz No. 445, Miraflores, Lima, Perú.

Teléfono: 422-9075

Correoelectrónico: alarcon.renato@mayo. edu 